\title{
A Diophantine problem on algebraic curves over function fields of positive characteristic
}

\author{
Bulletin de la S. M. F., tome 119, no 1 (1991), p. 121-126 \\ <http://www.numdam.org/item?id=BSMF_1991_119_1_121_0>
}

(C) Bulletin de la S. M. F., 1991, tous droits réservés.

L'accès aux archives de la revue «Bulletin de la S. M. F. » (http: //smf.emath.fr/Publications/Bulletin/Presentation.html) implique l'accord avec les conditions générales d'utilisation (http://www.numdam.org/ conditions). Toute utilisation commerciale ou impression systématique est constitutive d'une infraction pénale. Toute copie ou impression de ce fichier doit contenir la présente mention de copyright.

\section{NumDam}




\title{
A DIOPHANTINE PROBLEM ON ALGEBRAIC CURVES OVER FUNCTION FIELDS OF POSITIVE CHARACTERISTIC
}

\author{
BY \\ J.F. VOLOCH $(*)$
}

\begin{abstract}
RÉSumÉ. - Soit $K$ un corps de fonctions d'une variable sur un corps fini de caractéristique $p$. On détermine les courbes algébriques sur $K$ ayant une fonction $K$-rationnelle dont leurs valeurs dans une infinité de points $K$-rationnels sont des puissances $p$-èmes. On en déduit la finitude de l'ensemble des points rationnels des courbes sur $K$ qui changent de genre sous une extension de corps de base.

ABstract. - Let $K$ be a function field in one variable over a finite field of characteristic $p$. We determine the algebraic curves over $K$ having a $K$-rational function on it whose value at infinitely many $K$-rational points is a $p$-th power. From this we deduce the finiteness of the set of $K$-rational points of curves over $K$ that change genus under ground-field extension.
\end{abstract}

\section{Introduction}

Let $K$ be a function field in one variable over a finite field of characteristic $p$. The purpose of this paper is to characterize the algebraic curves $X / K$ and the rational functions $f \in K(X)$ such that $f(P) \in K^{p}$ for infinitely many rational points $P \in X(K)$. This problem ties up with a question left open by SAMUEL [2] in his extension to positive characteristic of GRAuERT's proof of MORDELL's conjecture for function fields of characteristic zero. The question occurs when the relative genus of $X / K$ is different from the absolute genus of $X$ in the sense of [2] (or equivalently when $K(X)$ is a non-conservative function field in the sense of [1]).

The genus of a curve $X$ defined over $K$, relative to $K$, can be defined as follows. It is the integer $g$ for which $\ell(D)=\operatorname{deg} D+1-g$, for divisors $D$, defined over $K$, with degree $\operatorname{deg} D$ sufficiently large, where $\ell(D)$ is the dimension, as a $K$-vector space, of the space of rational functions on $X$,

$\left.{ }^{*}\right)$ Texte reçu le 4 mai 1990, révisé le 13 septembre 1990.

J.F. VOLOCH, I.M.P.A., Estrada Dona Castorina 110, Rio de Janeiro 22460, Brésil.

BULLETIN DE LA SOCIÉtÉ MATHÉmATIQUE DE FRANCE 0037-9484/1991/121/\$5.00

(C) Société mathématique de France 
defined over $K$, with polar divisor bounded by $D$. The above definition of the genus depends on $K$. The genus of $X$, relative to $K$, does not change under separable extensions of $K$ but may decrease under inseparable extensions. The absolute genus of $X$ is thus defined as the genus of $X$ relative to the algebraic closure of $K$. A standard example, for $p \geq 3$, is the curve $y^{2}=x^{p}-a$. If $a \in K \backslash K^{p}$, then its genus, relative to $K$, is $\frac{1}{2}(p-1)$ and its absolute genus is 0 .

SAmuel showed that, with notation as above, $X(K)$ is finite if the absolute genus of $X$ is at least two [2, Chapitre III, Theorem 1 and app. 2] and therefore the problem above is trivial for those curves. The question left open by SAMUEL [2, page 3] is whether curves with relative genus at least two and absolute genus 0 or 1 have finitely many rational points and we solve this question in the affirmative. Note that we have shown previously [4] that curves with relative genus 1 and absolute genus 0 have finitely many rational points (this will also follow from THEOREM 1 below). Hence all curves that admit genus change have finitely many rational points.

The paper is organized as follows. In sections 2 and 3 we solve our basic problem for rational curves and elliptic curves, respectively, and in section 4 we use these results to show that curves that admit genus change have finitely many rational points. Finally, we obtain the general solution to our problem.

\section{Rational curves}

Recall that $K$ is a function field in one variable over a finite field of characteristic $p$. Let $t \in K \backslash K^{p}$ and $\delta=\mathrm{d} / \mathrm{d} t$, a derivation of $K$. If $x$ is a variable over $K$, we extend $\delta$ to $K(x)$ by $\delta(x)=0$. We shall also use the notation $r^{\delta}(x)$ for the action of $\delta$ on $r(x) \in K(x)$.

TheOREM 1. - Let $r(x) \in K(x)$ be a rational function such that the set $\left\{a \in K \mid r(a) \in K^{p}\right\}$ is infinite. Then, there exists $\left(\begin{array}{ll}\alpha & \beta \\ \gamma & \delta\end{array}\right) \in G L_{2}(K)$ such that $r((\alpha x+\beta) /(\gamma x+\delta)) \in K^{p}(x)$.

Proof. - Multiplying, if necessary, $r(x)$ by the $p$-th power of its denominator, we can assume that $r(x)$ is a polynomial. Let $n$ be the degree of $r(x)$ and assume first that $p \nmid n$.

Let $r(x)=a_{0} x^{n}+a_{1} x^{n-1}+\cdots+a_{n}$. By changing, if necessary, the variable $x$ to $a_{0}^{m} x$, where $m n+1 \equiv 0(p)$, we can assume that $a_{0} \in K^{p}$. Further, dividing $r(x)$ by $a_{0}$, we can also assume that $a_{0}=1$. Finally, changing $x$ to $x-a_{1} / n$, we can assume that $a_{1}=0$.

TOME $119-1991-\mathrm{N}^{\circ} 1$ 
If $a \in K$ is such that $r(a) \in K^{p}$, then

$$
0=\delta(r(a))=r^{\prime}(a) \delta a+r^{\delta}(a) .
$$

Note that $r^{\delta}(a)=\delta a_{2} x^{n-2}+\cdots+\delta a_{n}$ is of degree at most $(n-2)$. If $r^{\delta}(x)$ is identically zero, then $r(x) \in K^{p}[x]$, as desired. Assume then that $r^{\delta}(x) \neq 0$.

Let $v$ be a place of $K$ with $v\left(a_{i}\right) \geq 0, i=0, \ldots, n$ and $v(\mathrm{~d} t)=0$. If $a \in K$ is such that $v(a)<0$ then, clearly, $v\left(r^{\delta}(a)\right) \geq(n-2) v(a)$ and $v\left(r^{\prime}(a)\right)=(n-1) v(a)$, whence $v(\delta a) \geq 0$, from $\left(^{*}\right)$. If $v(a) \geq 0$ then, obviously, $v(\delta a) \geq 0$, as well. Thus $v(\delta a) \geq 0$ for all but finitely many places of $K$.

Further, the rational function $-r^{\delta}(x) / r^{\prime}(x)$ has a zero at infinity. Thus, for any place $v$ of $K$, if $a$ has a sufficiently large pole at 0 then $\delta a=-r^{\delta}(a) / r^{\prime}(a)$ satisfies $v(\delta a) \geq 0$, say. On the other hand, if $v(a)$ is bounded below, then $v(\delta a)$ is also bounded below. The conclusion of the above discussion is that there exists a divisor $D$ of $K$ such that $\delta a \in L(D)$ for any $a \in K$ with $r(a) \in K^{p}$. Hence, $\delta a$ can assume finitely many values $b_{1}, \ldots, b_{N}$ for those $a$. The polynomial equations $r^{\prime}(x) b_{i}+r^{\delta}(x)=0, i=1, \ldots, N$, have finitely many solutions unless one of them is identically zero. In the latter case, looking at the coefficient in $x^{n-1}$, it follows that $b_{i}=0$ (recall that $p+n$ ) and so $r^{\delta}(x)=0$, contrary to the hypothesis. This proves the result when $p \nmid n$.

Let now $r(x)$ be a polynomial of degree $n \equiv 0(p)$ satisfying the hypothesis of the theorem. Let $a \in K$ be such that $r(a) \in K^{p}$. To prove the theorem for $r(x)$ it suffices to prove the theorem for the polynomial $x^{n}(r(1 / x+a)-r(a))$, which has degree strictly less than $n$. The theorem now follows by induction on $n$.

REMARK 1. - Let $r(x) \in K(x)$ be such that there exists $\left(\begin{array}{cc}\alpha & \beta \\ \gamma & \delta\end{array}\right) \in$ $G L_{2}(K)$ with $r((\alpha x+\beta) /(\gamma x+\delta)) \in K^{p}(x)$. Then $r(a) \in K^{p}$ for infinitely many $a \in K$. Indeed $r\left(\left(\alpha x^{p}+\beta\right) /\left(\gamma x^{p}+\delta\right)\right)=(s(x))^{p}$ for some $s(x) \in K(x)$. This also shows that the curve $y^{p}=r(x)$ is parametrizable over $K$, that is, has relative genus zero over $K$.

Remark 2. - Theorem 1 contains, as special cases, the results of [4]. The proof of THEOREM 1 is an extension of the techniques of [4]. 


\section{Elliptic curves}

We keep the notation of section 2. In particular, recall the derivation $\delta$ of $K$. If $E / K$ is an elliptic curve given by the Weierstrass equation $y^{2}+a_{1} x y+a_{3} y=x^{3}+a_{2} x^{2}+a_{4} x+a_{6}$, let $E^{(p)} / K$ be the elliptic curve with Weierstrass equation $y^{2}+a_{1}^{p} x y+a_{3}^{p} y=x^{3}+a_{2}^{p} x^{2}+a_{4}^{p} x+a_{6}^{p}$ and $F: E \rightarrow E^{(p)}$ be the Frobenius map defined by $F(x, y)=\left(x^{p}, y^{p}\right)$. Let also $V: E^{(p)} \rightarrow E$ be the isogeny dual to $F$. We extend $\delta$ to a derivation on $K\left(E^{(p)}\right)=K(x, y)$ by $\delta(x)=\delta(y)=0$. As in section 2 we also denote by $r^{\delta}$ the action of $\delta$ on $r \in K\left(E^{(p)}\right)$.

THEOREM 2. - Notation as above. If $r \in K\left(E^{(p)}\right)$ is such that the set $\left\{P \in E^{(p)}(K) \mid r(P) \in K^{p}\right\}$ is infinite, then there exists $P_{0} \in E^{(p)}(K)$ such that the function $P \mapsto r\left(P+P_{0}\right)$ belongs to $K^{p}\left(E^{(p)}\right)$. If $r \in K(E)$ is such that the set $\left\{P \in E(K) \mid r(P) \in K^{p}\right\}$ is infinite, then there exists $P_{0} \in E(K)$ such that the function $P \mapsto r\left(V(P)+P_{0}\right)$ belongs to $K^{p}\left(E^{(p)}\right)$.

Proof. - Let $r \in K\left(E^{(p)}\right)$ satisfy the hypothesis of the theorem. As $E^{(p)}(K) / F(E(K))$ is finite (by the Mordell-Weil theorem) it follows that there exists $P_{0} \in E^{(p)}(K)$ such that, for infinitely many $P \in F(E(K))$, $r\left(P+P_{0}\right) \in K^{p}$. Let $s \in K\left(E^{(p)}\right)$ be defined by $s(P)=r\left(P+P_{0}\right)$. If $P \in F(E(K))$, its $x, y$ coordinates are $p$-th powers, hence $\delta(s(P))=$ $s^{\delta}(P)$. If, furthermore, $s(P) \in K^{p}$ then $s^{\delta}(P)=0$. But $s^{\delta}$ has finitely many zeros unless is identically zero. We therefore conclude that $s^{\delta}=0$, that is, $s \in K^{p}\left(E^{(p)}\right)$, as desired.

Let $r \in K(E)$ satisfy the hypothesis of the theorem. Again by MordellWeil, $E(K) / V\left(E^{(p)}(K)\right)$ is finite : there exists $P_{1} \in E(K)$ such that there exists infinitely many $P \in V\left(E^{(p)}(K)\right)$ with $r\left(P+P_{1}\right) \in K^{p}$. Thus, the function $P \mapsto r\left(V(P)+P_{1}\right)$ on $E^{(p)}$, satisfies the hypothesis of the theorem and, by what was proved above, there exists $P_{2}$ such that the function $P \mapsto r\left(V\left(P+P_{2}\right)+P_{1}\right)$ belongs to $K^{p}\left(E^{(p)}\right)$ and the theorem follows with $P_{0}=P_{1}+V\left(P_{2}\right)$.

REMARK 3. - If $r \in K\left(E^{(p)}\right)$ is such that $P \mapsto r\left(P+P_{0}\right)$ belongs to $K^{p}\left(E^{(p)}\right)$ for some $P_{0} \in E^{(p)}(K)$ then $r(P) \in K^{p}$ for all $P \in E^{(p)}(K)$, $P-P_{0} \in F(E(K))$. Indeed $r\left(F(P)+P_{0}\right)=(s(P))^{p}$ for some $s \in K(E)$. Thus the cover of $E^{(p)}$ defined by the equation $z^{p}=r$ has genus 1 over $K$, since it is covered by $E$ by the map $P \mapsto\left(F(P)+P_{0}, s(P)\right)$. A similar phenomenon occurs for $r \in K(E)$ such that $P \mapsto r\left(V(P)+P_{0}\right)$ belongs to $K^{p}\left(E^{(p)}\right)$. Indeed, $r\left(p P+P_{0}\right)=s(P)^{p}$ for some $s \in K(E)$, since $V \circ F$ is multiplication by $p$ on $E$.

TOME $119-1991-\mathrm{N}^{\circ} 1$ 


\section{Mordell's conjecture for non-conservative curves}

An algebraic curve $X / K$ is said to be conservative if its genus does not change under base-field extension from $K$ to its algebraic closure $\bar{K}$ (see [2, page 3] or [1], [3]). Otherwise $X$ is called non-conservative. The genus of $X$ over $K$ is called the relative genus of $X$ and the genus of $X$ over $\bar{K}$, the absolute genus of $X$.

We retain the notation as above, in particular $K$ is a function field in one variable over a finite field.

Theorem 3. - A non-conservative algebraic curve $X / K$ has finitely many $K$-rational points.

Proof. - Let $X_{n} / K, n=0,1, \ldots$ be the algebraic curve whose function field is $K \cdot(K(X))^{p^{n}}$ and denote by $g_{n}$ the genus of $X_{n}$.

The sequence $g_{n}$ is non-increasing and the constant value of $g_{n}$ for all $n$ sufficiently large is the absolute genus $\bar{g}$ of $X$ (see $e . g$. [3]). If $\bar{g} \geq 2$, then the theorem was already proved by SAmuel. Assume that $\bar{g}=0$ or 1 and let $n$ be such that $g_{n-1}>g_{n}=\bar{g}$. Let $z \in K\left(X_{n-1}\right) \backslash K\left(X_{n}\right)$, then $r=z^{p} \in K\left(X_{n}\right)$ and $K\left(X_{n-1}\right)=K\left(X_{n}\right)(z)$. This means that $X_{n-1}$ is the cover of $X_{n}$ given by $z^{p}=r$ and it is easy to see that all but finitely many rational points of $X_{n-1}$ correspond to rational points $P \in X_{n}$ for which $r(P) \in K^{p}$. From Theorem 1 (and Remark 1) and Theorem 2 (and REMARK 3 ) it follows that there exists only finitely many such points and thus $X_{n-1}(K)$ is finite. It then follows by a similar and easier argument that $X_{n-2}(K), \ldots, X_{0}(K)=X(K)$ are all finite, proving the theorem.

REMARK 4. - Returning to our basic problem, staded in the introduction, if $X / K$ is an algebraic curve and $f \in K(X)$ is such that $\left\{P \in K(K) \mid f(P) \in K^{p}\right\}$ is infinite, then by ThEorem 3 and the GrauertSamuel Theorem (i.e. the Mordell conjecture in characteristic $p$ ) it follows that $X$ is rational or elliptic, and in these cases the problem is solved by TheOREMS 1 and 2 .

\section{BIBLIOGRAPHY}

[1] Artin (E.).- - Algebraic Numbers and Algebraic Functions. - Gordon and Bleach, New York-London-Paris, 1967. 
[2] Samuel (P.). - Lectures on old and new results on Algebraic Curves. - Tata Institute of Fundamental Research, Bombay, 1966.

[3] Stichtenoth (H.). - Zur Konservativität algebraischer Funktionenkörper, Crelle, t. 301, 1978, p. 30-45.

[4] Voloch (J.F.). - Mordell's equation in characteristic three, Bull. Austral. Math. Soc., t. 41, 1990, p. 149-150. 